\section{Intraocular lens power calculation in short eyes}

EA Gavin and CJ Hammond

\author{
EA Gavn and
} in

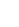

\begin{abstract}
Purpose To compare the accuracy of the Hoffer $Q$ and SRK-T formulae in eyes below $22 \mathrm{~mm}$ in axial length, using biometry measured with partial coherence inferometry (PCI), without a customised ACD constant. Methods Data were retrospectively and prospectively collected by identifying eyes of axial length below $22 \mathrm{~mm}$ in the records of the intraocular lens (IOL) master and in preoperative notes. Biometry was performed using PCI and IOL power was calculated using both SRK-T and Hoffer $Q$ formulae. Refractive outcome was measured and the accuracy of the two formulae compared.
\end{abstract}

Results Forty-one eyes of 41 patients were identified with an axial length $<22 \mathrm{~mm}$. Axial lengths ranged from 21.96 to 20.29 with a mean of $21.51 \mathrm{~mm}$, and IOL power ranging from 23 dioptres (D) to $29 \mathrm{D}$. The Hoffer $Q$ formula showed a mean prediction error of $0.61 \mathrm{D}$ (SD 0.80) compared with the SRK-T, which showed a mean prediction error of $0.87 \mathrm{D}$ (SD 0.829). A paired $t$-test found that the Hoffer $Q$ was significantly more accurate than the SRK-T formula $(P<0.001)$.

Conclusions Hoffer $Q$ was found to be more accurate than the SRK-T formula in this series of eyes $<22 \mathrm{~mm}$ axial length when customised ACD constants are not used. Royal College of Ophthalmologists guidelines may need to be adjusted in accordance with these findings. This study underlines the importance of monitoring outcomes, and suggests different customisations are needed for different formulae, with a higher correction if the SRK-T formula is used for short eyes.

Eye (2008) 22, 935-938; doi:10.1038/sj.eye.6702774; published online 16 March 2007

Keywords: cataract; hypermetropia; biometry; lens implantation; cataract extraction; phacoemulsification

\section{Introduction}

Intraocular lens (IOL) power calculation accuracy has been of great importance in cataract surgery for many years but with an increasing demand for low postoperative refractive error and increasingly high patient expectations, the need for accurate power calculation is paramount. In the past two decades, there have been advances in both surgical technique and in the accuracy of biometric measurements. The routine use of phacoemulsification and sutureless incisions combined with continuous curvilinear capsulorhexis has improved the predictability of refractive outcome. ${ }^{1}$ In addition, optical biometry with partial coherence inferometry (PCI) has been shown to yield significantly better IOL power prediction, and therefore refractive outcome in cataract surgery, than ultrasound biometry. ${ }^{2}$ Improvements in measurement and surgical technique have raised a debate about the best IOL calculation formula to obtain the most precise prediction of refractive outcome.

The 2001 Royal College of Ophthalmologists (RCOphth) Cataract Surgery Guidelines issued recommendations on biometry and IOL calculation according to the axial length of the eye, ${ }^{3}$ shown in Table 1 . These guidelines were based on Hoffer's recommendations, ${ }^{4}$ and did not discuss A constant customisation, which had been recommended by Hoffer. The National Biometry audit, published in 2004, found poor adherence to these guidelines, with only $36 \%$ of departments using two or more formulae, and only $4 \%$ using the Hoffer $Q$ formula in eyes with axial lengths below $22 \mathrm{~mm} .{ }^{5}$ The current 2004 RCOphth Guidelines advise use of Hoffer- $\mathrm{Q}^{4}$ or SRK-T ${ }^{6}$ formulae for eyes $<22 \mathrm{~mm}$ length, as well as use and checking of the appropriate ACD constants. ${ }^{7}$

Hoffer's original series ${ }^{4}$ included 36 eyes below $22 \mathrm{~mm}$ in axial length, and suggested the Hoffer $Q$ may be more accurate than the SRK-T (not statistically significant, $P=0.83$, probably
West Kent Eye Centre, Bromley Hospitals NHS Trust, Orpington, UK

Correspondence:

CJ Hammond,

West Kent Eye Centre, Princess Royal University Hospital,

Farnborough Common, Orpington, Kent BR6 8ND, UK

Tel: + 4401689863000 ;

Fax: + 4401689863329

E-mail: chammond@

btopenworld.com

Received: 18 September 2006

Accepted in revised form: 11 February 2007 Published online: 16 March 2007 
due to the relatively small size of the series). Hoffer also investigated the accuracy of the Hoffer $\mathrm{Q}$ against the SRK-T again in $2000^{8}$ (in addition to the Holladay 1 and Holladay 2 formulae) and again the Hoffer $Q$ appeared more accurate than the SRK-T, but with only 10 eyes in the series below $22 \mathrm{~mm}$ in axial length, no statistical significance was tested.

The purpose of this study was to compare the accuracy of the Hoffer $\mathrm{Q}$ and SRK-T formulae in eyes below $22 \mathrm{~mm}$ in axial length, using biometry measured with PCI, without a customised ACD constant, to ascertain whether one formula was more accurate than the other.

\section{Materials and methods}

Data were collected retrospectively and prospectively by identifying eyes of patients undergoing routine cataract surgery with an axial length below $22 \mathrm{~mm}$ from the biometry database and in preoperative notes. Biometry was performed using PCI (IOLMaster, Zeiss Humphrey Systems) and IOL power was calculated using the SRK-T formula and the manufacturer's A constant. In addition, the Hoffer Q formula was calculated with the same biometric data for each eye.

Patients underwent routine clear-corneal phacoemulsification and Allergan MA60 foldable IOL insertion into the capsular bag. Following uneventful surgery, patients were reviewed in the eye clinic 2-3 weeks

Table 1 Royal College guidelines on formula usage according to axial length interval

\begin{tabular}{lll}
\hline Axial length $(\mathrm{mm})$ & $\begin{array}{l}\text { RCOphth 2001 } \\
\text { Guideline }\end{array}$ & $\begin{array}{l}\text { RCOphth 2004 } \\
\text { Guideline }\end{array}$ \\
\hline$<22$ & Hoffer Q & Hoffer Q or SRK-T \\
$22.0-24.5$ & Average Hoffer Q & Hoffer Q, Haigis or \\
& Holladay and SRK-T & SRK-T \\
$24.6-26.0$ & Holladay & SRK-T \\
$>26.0$ & SRK-T & SRK-T \\
\hline
\end{tabular}

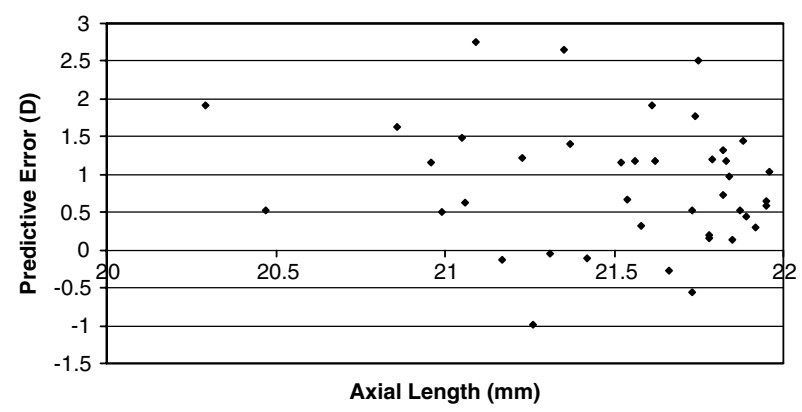

Figure 1 Predictive error (in dioptric spherical equivalent) in a series of 41 short eyes ( $<22 \mathrm{~mm}$ axial length), using biometry obtained by PCI and the SRK-T formula. following surgery. Objective measurement of the refractive status of the eye was obtained with the autorefractor (Canon AR-2, Canon, Tokyo) and this was converted into a spherical equivalent value, which was taken as the refractive outcome. The predictive errors were calculated for both formulas - the predictive error was defined as the difference in the predicted value from the biometry for each formula and the spherical equivalent value of the postoperative objective refraction. The accuracy of the two methods was compared using a paired $t$-test.

\section{Results}

Patients undergoing routine cataract surgery at our hospital between April 2004 and March 2005 were identified. Forty-six patients undergoing cataract surgery with axial length $<22 \mathrm{~mm}$ were identified and of these five were excluded: the notes/biometry/postoperative autorefraction were incomplete for three patients, one patient's biometry related to a lens exchange, and one patient developed postoperative endophthalmitis. In total, 41 eyes of 41 patients were included. Axial lengths ranged from 20.29 to $21.96 \mathrm{~mm}$, with a mean of $21.51 \mathrm{~mm}$, and the IOL power inserted ranged from 23 dioptres (D) to $29 \mathrm{D}$ (mean $25.73 \mathrm{D}$, median 26 D). The planned refraction aimed for was determined by individual surgeons, based on the preoperative biometry (using the SRK-T formula and manufacturer's A constant).

Comparison of actual outcomes with predicted outcomes using PCI biometry and IOL calculation formulae, without any correction/A constant customisation, resulted in a more hypermetropic outcome than predicted for both formulae. Figure 1 shows the distribution of the predictive errors in this series using the SRK-T formula. The Hoffer Q formula's mean prediction error of $0.61 \mathrm{D}$ was lower than the mean prediction error of the SRK-T formula $(0.87 \mathrm{D})$, shown in Table 2. Table 2 also shows the ranges for the two formulae, with the Hoffer $Q$ having a higher proportion of patients achieving $+/-1 \mathrm{D}$ of the predicted outcome than the SRK-T (63 vs 54\%). A paired $t$-test found that the Hoffer $Q$ was significantly more accurate than the SRK-T formula $(P<0.001)$.

If a customised correction factor for each formula was applied ( + 0.61 D for Hoffer Q and + 0.87 D for SRK-T added to the manufacturer's A constant), then the two formulae resulted in similar outcomes, with $80 \%$ of subjects within $1 \mathrm{D}$ of the predicted value for the SRK-T and $83 \%$ for the Hoffer Q, as shown in Table 3.

\section{Discussion}

To our knowledge this series is the first to show the Hoffer $Q$ formula is statistically significantly more 
Table 2 Predictive errors and predictive ranges for the SRK-T and Hoffer Q formulae

\begin{tabular}{lll}
\hline & SRK-T & Hoffer $Q$ \\
\hline Mean (SD) error & $0.87(0.829)$ & $0.61(0.80)$ \\
Mean absolute (SD) error & $0.98(0.701)$ & $0.78(0.654)$ \\
\pm 0.25 D of predicted value & $17 \%$ & $27 \%$ \\
\pm 0.5 D of predicted value & $24 \%$ & $44 \%$ \\
\pm 1.0 D of predicted value & $54 \%$ & $63 \%$ \\
\hline
\end{tabular}

Table 3 Predictive ranges for the SRK-T and Hoffer Q formulae with corrective factor adjustment.

\begin{tabular}{lll}
\hline $\begin{array}{l}\text { Formula and 'Correction } \\
\text { Factor' }\end{array}$ & $\begin{array}{l}\text { SRK- } \\
T+0.87 D\end{array}$ & $\begin{array}{l}\text { Hoffer } \\
Q+0.61 D\end{array}$ \\
\hline $\pm 0.25 \mathrm{D}$ of predicted value & $12 \%$ & $15 \%$ \\
$\pm 0.5 \mathrm{D}$ of predicted value & $49 \%$ & $44 \%$ \\
$\pm 1 \mathrm{D}$ of predicted value & $80 \%$ & $83 \%$ \\
\hline
\end{tabular}

accurate than the SRK-T, and is also the largest series investigating biometry accuracy in eyes below $22 \mathrm{~mm}$ in axial length. Our results also indicate that the Hoffer $Q$ is more accurate than the SRK-T when customised ACD constants are not used, which was the case for $53 \%$ of departments in the UK in the second National Biometry Audit. ${ }^{9}$ The SRK-T formula was developed to increase accuracy in high myopia ${ }^{6}$ and the Hoffer $Q$ formula for use in extreme hyperopia, ${ }^{4}$ and this study confirms the previous impression that the Hoffer $Q$ is more accurate than the SRK-T for this group of patients. Although current Royal College guidelines allow the SRK-T to be used for all axial lengths, these results highlight the need to audit the refractive outcomes of myopes,

hypermetropes, and emmertropes separately.

Use of the SRK-T formula tended to result in a more hypermetropic refraction than the Hoffer Q. However, if a different correction factor was applied $(0.61 \mathrm{D}$ for the Hoffer $\mathrm{Q}$ and 0.87 for the SRK-T), then the results of the two formulae are comparable (Table 3). These results emphasise the importance of individual surgeons and departments to closely audit their results, and to adopt customised A constants depending on the formula used and previous outcomes. Based on our results, a greater correction than normal is required in hyperopic eyes to achieve emmetropia, particularly if the SRK-T formula is used.

Any measurement error in the axial length of a short eye will have a larger effect on the final refraction, as a given measurement error is a much larger percentage of the axial length in a short eye and hence this could be an important source of error. This is of particular importance in applanation ultrasound biometry, as this method is known to have the disadvantage of potentially depressing the cornea during use leading to a falsely short axial length measurement. The use of the PCI in this study should have minimised biometry errors as this method has a higher resolution than standard ultrasound transducers, ${ }^{10,11}$ is highly reproducible ${ }^{12}$ and avoids any contact with the eye.

The prospective study of 1817 eyes of all axial lengths by Murphy et $\mathrm{al}^{13}$ achieved a final refraction within $1 \mathrm{D}$ of the biometry-predicted value in $72.3 \%$ of subjects, and may be considered a benchmark for current cataract practice and a useful audit standard. However, Murphy et al did not state the proportion of eyes below $22 \mathrm{~mm}$ or the biometry prediction error for this subgroup, and therefore comparison between the current study and their data is not valid.

A potential source of error in this study was the use of the autorefractor as the method of measuring the spherical equivalent for postoperative outcome. Other studies have found there is no significant difference in the accuracy of the autorefractor and subjective refraction in assessment of the final achieved refractive outcome for LASIK $^{14}$ and cataract surgery, ${ }^{15}$ indicating that the autorefractor is an acceptable outcome measure of final refractive status. The advantage of use of the autorefractor measure to be able to include all patients with axial length $<22 \mathrm{~mm}$ outweighed the disadvantage of trying to rely on later (possibly more accurate) refraction from optometrists, with the likelihood of incomplete data collection and possible ascertainment bias.

In conclusion, the Hoffer $\mathrm{Q}$ was found to be more accurate than the SRK-T formula with statistical significance in this series of eyes $<22 \mathrm{~mm}$ axial length. To our knowledge, this has not been reported previously, and this is the largest series specifically reporting the refractive outcomes of short eyes in cataract surgery. RCOphth Guidelines may need to be adjusted in accordance with these findings; failing this, surgeons may need to factor a different (higher) customisation constant for eyes of small axial length, depending on the formula used, and to audit their own results in this group of patients.

\section{Acknowledgements}

This work has previously been presented as a poster at The Royal College of Ophthalmologists Congress, Manchester, May 2006.

\section{References}

1 Minassian DC. Extracapsular cataract extraction compared with small incision surgery by phacoemulsification: a randomised trial. Br J Ophthalmol 2001; 85(7): 882-889. 
2 Findl O, Drexler W, Menapace R, Heinzl H, Hitzenberger $\mathrm{CK}$, Fercher AF. Improved prediction of intraocular lens power using partial coherence interferometry. J Cataract Refract Surg 2001; 27: 861-867.

3 The Royal College of Ophthalmologists. Cataract Surgery Guidelines. www.rcophth.ac.uk,February 2001.

4 Hoffer KJ. The Hoffer Q formula: a comparison of theoretic and regression formulas. J Cataract Refract Surg 1993; 19: 700-712.

5 Gale RP, Saha N, Johnston RL. National Biometry Audit. Eye 2004; 18: 63-66.

6 Retzlaff JA, Sanders DR, Kraff MC. Development of the SRK-T intraocular lens implant power calculation formula. J Cataract Refract Surg 1990; 16: 333-340.

7 The Royal College of Ophthalmologists. Cataract Surgery Guidelines. www.rcophth.ac.uk,March 2004.

8 Hoffer KJ. Clinical results using the Holladay 2 intraocular lens power formula. J Cataract Refract Surg 2000; 26: 1233-1237.

9 Gale RP, Saha N, Johnston RL. National Biometry Audit II. Eye 2006; 20: 25-28.
10 Findl O, Drexler W, Menapacev R, Hitzenberger CG, Fercher AF. High precision biometry of pseudophakic eyes using partial coherence interferometry. J Cataract Surg 1998; 24: 1087-1093.

11 Schachar RA, Levy NS, Bonney RC. Accuracy of intraocular lens powers calculated from A scan biometry with the Echo-oculometer. Ophthalmic Surg 1980; 11: 856-858.

12 Connors R, Boseman P, Olson R. Accuracy and reproducibility of biometry using partial coherence interferometry. J Cataract Refract Surg 2002; 28: 235-238.

13 Murphy C, Tuft SJ, Minassian DC. Refractive error and visual outcome after cataract extraction. J Cataract Refract Surg 2002; 28: 62-66.

14 Pesudovs K. Autorefraction as an outcome measure of laser in situ keratomileusis. J Cataract Refract Surg 2004; 30: 1921-1928.

15 Gavin E, Hove M, Goel R, Gibbens M. The accuracy of the autorefractor as an assessment of final refractive outcome following routine phacoemulsification. Proceedings of 10th ESCRS Refractive Surgery Meeting, Monte Carlo, 10-12th February, Book of Abstracts, p 34 